phys. stat. sol (b) 215, 33 (1999)

Subject classification: 78.20.Ci; 71.35.Cc; S7.12; S7.13; S8.13

\title{
Modeling the Optical Constants of Diamond- and Zincblende-Type Semiconductors: Discrete and Continuum Exciton Effects at $\mathbf{E}_{0}$ and $\mathbf{E}_{1}$
}

\author{
F.H. PollaK ${ }^{1}$ ), M. Muñoz ${ }^{2}$ ), T. Holden, K. Wei, and V. M. Asnin \\ Physics Department and NY State Center for Advanced Technology in Ultrafast Photonic \\ Materials and Applications, Brooklyn College of CUNY, Brooklyn, NY 11210, USA
}

(Received March 30, 1999)

We present a comprehensive model dielectric function $\varepsilon(E)\left[=\varepsilon_{1}+i \varepsilon_{2}\right]$ for diamond- and zincblende-type semiconductors based on the energy-band structure near critical points (CPs) plus discrete as well as continuum excitonic effects at the $E_{0}, E_{0}+\Delta_{0}, E_{1}$, and $E_{1}+\Delta_{1}$ CPs. In addition to the energies of these band-to-band CPs, our analysis also yields information about the binding energies of not only the 3D exciton associated with $E_{0}\left(R_{0}\right)$, when resolved, but also the $2 \mathrm{D}$ exciton related to the $E_{1}, E_{1}+\Delta_{1}$ CPs $\left(R_{1}\right)$. This model has been applied to spectral ellipsometry measurements of $\varepsilon_{1}, \varepsilon_{2}(0.3 \mathrm{eV}<E<5.5 \mathrm{eV})$ of $\mathrm{ZnCdSe} / \mathrm{InP}, \mathrm{CdTe}_{1-x} \mathrm{~S}_{x}, \mathrm{In}_{0.66} \mathrm{Ga}_{0.34} \mathrm{As}$, and $\mathrm{GaSb}$ and a surface photovoltage spectroscopy determination of the absorption coefficient of GaAs near $E_{0}$. This work shows conclusively that even if the exciton at $E_{0}$ is not resolved the lineshape is continuum exciton. The obtained values of $R_{1}$ exhibit a trend which is in good agreement with effective mass $/ \mathbf{k} \cdot \mathbf{p}$ theory. Our analysis will be compared with the modeling of Adachi and the University of Illinois-Chicago group, both of whom neglect exciton continuum effects and hence have not evaluated $R_{1}$. Our results, particularly for exciton continuum effects at $E_{1}$, have considerable implications for recent first-principles band structure calculations which include exciton effects.

The optical constants associated with electronic transitions of diamond- and-zincblendetype (DZB) materials have been measured by a number of methods. During the past decade spectral ellipsometry (SE) has been used by number of investigators including Aspnes and Studna [1], Cardona and coworkers [2,3], Adachi and coworkers [4], the University of Illinois-Chicago group [5], etc. However, these workers either did not model the optical constants [1 to 3] or used an incomplete single particle expression $[4,5]$. Cardona and coworkers fit derivative (with respect to photon energy) spectra employing a two-dimensional density of states for the " $E_{1}, E_{1}+\Delta_{1}$ " features; exciton effects were taken into account by means of a phase angle (Slater-Koster "contact" potential) [2,3]. Recently, Holden et al. [6] have presented a comprehensive model dielectric function $\varepsilon(E)\left[=\varepsilon_{1}+i \varepsilon_{2}\right]$ for DZB semiconductors based on the energy-band structure near critical points (CPs) plus discrete as well as band-to-band Coulomb enhanced (BBCE) effects, i.e., continuum excitons, at the $E_{0}, E_{0}+\Delta_{0}, E_{1}$, and $E_{1}+\Delta_{1}$ CPs. In addition to the energies of these band-to-band CPs, this analysis also yields information about the binding energies of not only the 3D exciton associated with

\footnotetext{
1) Corresponding author: Tel.: 718/951-5356; Fax: 718/951-4871;

e-mail: FHPBC@CUNYVM.CUNY.EDU

$\left.{ }^{2}\right)$ Permanent address: Departamento de Fisica, CINVESTAV, Mexico DF, Mexico. e-mail: MARTIN@FIS.CINVESTAV.MX
} 
$E_{0}\left(R_{0}\right)$, when resolved, but also the $2 \mathrm{D}$ exciton related to the $E_{1}, E_{1}+\Delta_{1} \mathrm{CPs}\left(R_{1}\right)$. This model has already been applied to SE measurements of $\varepsilon_{1}, \varepsilon_{2}$ (energy range $0.3 \mathrm{eV}<E<5.5 \mathrm{eV}$ ) of $\mathrm{ZnCdSe} / \mathrm{InP}$ [6], $\mathrm{CdTe}_{1-x} \mathrm{~S}_{x}$ [7], and $\mathrm{In}_{0.66} \mathrm{Ga}_{0.34}$ As (undoped as well as $\mathrm{n}$ - and p-doped) [8].

In this paper we present an SE investigation of GaSb (in the photon energy range 0.3 to $5.3 \mathrm{eV}$ ) as well as a surface photovoltage spectroscopy (SPS) study of undoped GaAs near $E_{0}$. By fitting the SE data of GaSb to the Holden model we have evaluated $R_{1}$ in addition to the energies of the various CPs. The values of $R_{1}$ for $\mathrm{ZnCdSe} / \mathrm{InP}$, $\mathrm{CdS}, \mathrm{CdTe}, \mathrm{In}_{0.66} \mathrm{Ga}_{0.34} \mathrm{As}$, and $\mathrm{GaSb}$ exhibit a trend which is in good agreement with effective mass $/ \mathbf{k} \cdot \mathbf{p}$ theory [9]. The ability to determine $R_{1}$ has considerable implications for recent first-principles band structure calculations which include exciton effects [10]. An analysis of the GaAs SPS data (measured in this work) and the CdTe SE [8] results near $E_{0}$ show conclusively that even if the exciton at this $\mathrm{CP}$ is not resolved the lineshape is BBCE and not band-to-band single particle (BBSP) [4,5].

The sample consisted of bulk GaSb (001) (cut $4^{\circ}$ towards [110]). The optical data in the range 0.75 to $5.3 \mathrm{eV}$ were taken using an Instruments SA variable angle ellipsometer while for the interval 0.3 to $0.8 \mathrm{eV}$ a variable angle instrument which used a Fourier transform infrared reflectometer as a light source was employed. Thus there was some overlap between the two intervals. The SPS data were taken using a normalized Kelvin probe technique [11].

The solid line in Fig. 1 is the experimental imaginary component $\varepsilon_{2}(E)$ of the complex dielectric function for GaSb. The obtained $\varepsilon_{1}(E)$ spectrum is presented in Ref. [12]. Our data are very similar to that ones reported in Refs. $[1,2,13]$ in the appropriate energy ranges. References [1,2] made measurements only in the range 1.5 to $5.5 \mathrm{eV}$. There is an absorption edge around $0.7 \mathrm{eV}$, doublet peaks in the range 2.0 to $2.5 \mathrm{eV}$,

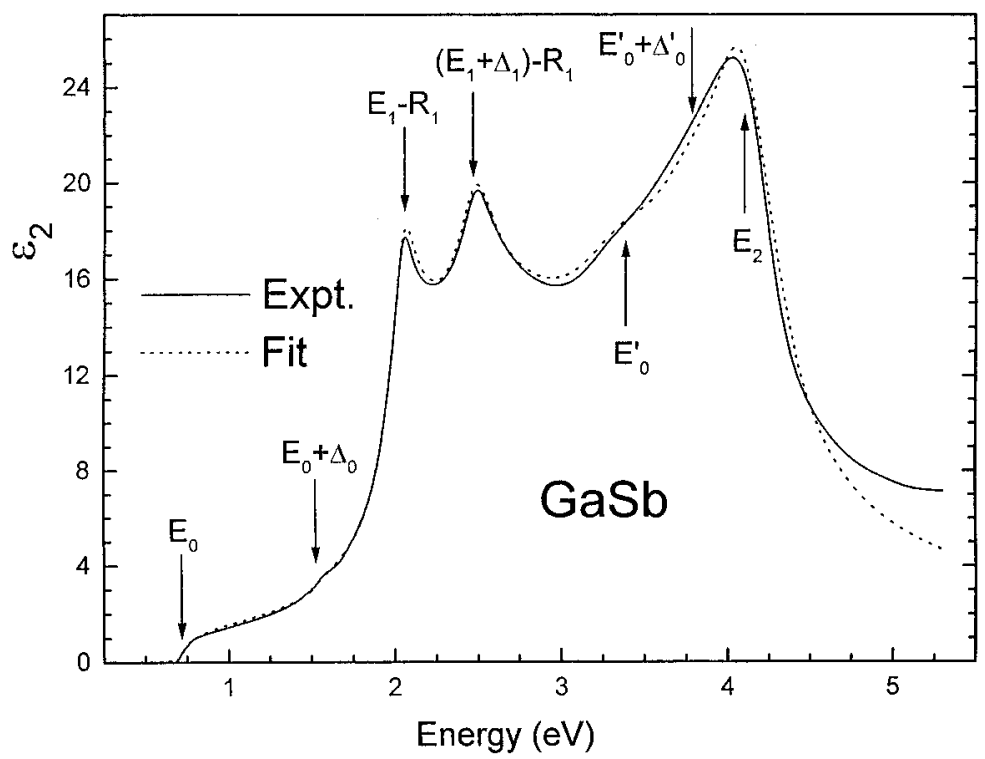

Fig. 1. The solid and dashed lines represent the experimental and fit values, respectively, of the imaginary component $\left(\varepsilon_{2}\right)$ of the complex dielectric function of $\mathrm{GaSb}$ 
and a large feature with a peak around $4 \mathrm{eV}$ with some structure on the low energy side around $3.5 \mathrm{eV}$. There is also a weak feature at around $1.5 \mathrm{eV}$, not observed in Refs. [1,2,13].

The data of Fig. 1 near the $E_{0}$ band gap has been fit to a function which contains Lorentzian broadened (a) discrete excitonic (DE) and (b) 3D $M_{0}$ BBCE contributions, i.e., continuum exciton effects. As will be demonstrated below, even if the $E_{0}$ exciton is not resolved, the Coulomb interaction still affects the band-to-band lineshape. Therefore we can write $[6$ to 8,12$]$

$$
\begin{aligned}
\varepsilon_{2}(E)= & \operatorname{Im}\left\{\frac { A } { E ^ { 2 } } \left\{\left[\frac{2 R_{0}}{\left(E_{0}-R_{0}\right)-E-i \Gamma_{0}^{\mathrm{ex}}}+\frac{2 R_{0}}{\left(E_{0}-R_{0}\right)+E+i \Gamma_{0}^{\mathrm{ex}}}\right]\right.\right. \\
& \left.\left.+\int_{-\infty}^{\infty}\left[\frac{\theta\left(E^{\prime}-E_{0}\right)}{1-\mathrm{e}^{-2 \pi z_{1}\left(E^{\prime}\right)}}-\frac{\theta\left(-E^{\prime}-E_{0}\right)}{1-\mathrm{e}^{-2 \pi z_{2}\left(E^{\prime}\right)}}\right] \frac{\mathrm{d}^{\prime} E}{E^{\prime}-E-i \Gamma_{0}}\right\}\right\},
\end{aligned}
$$

where $A$ is a constant, $E_{0}$ is the energy of the direct gap, $R_{0}$ is the effective Rydberg energy $\left[=\left(E_{0}-E_{0}^{\mathrm{ex}}\right)\right], \Gamma_{0}^{\mathrm{ex}}$ is the broadening of the exciton, $\Gamma_{0}$ is the broadening parameter for the BBCE transition, $z_{1}(E)=\left[R_{0} /\left(E-E_{0}\right)\right]^{1 / 2}, z_{2}(E)=\left[R_{0} /\left(-E-E_{0}\right)\right]^{1 / 2}$ and $\theta(x)$ is the unit step function. For the $E_{0}+\Delta_{0}$ transition, Eq. (1) will have $A \rightarrow B$, $E_{0} \rightarrow E_{0}+\Delta_{0}, R_{0} \rightarrow R_{\text {so }}$ and $\Gamma \rightarrow \Gamma_{\text {so. }}$

For $E_{1}$ we use [6 to 8,12 ]

$$
\begin{aligned}
\varepsilon_{2}(E)= & \operatorname{Im}\left\{\frac { C _ { 1 } } { E ^ { 2 } } \left\{\left[\frac{4 R_{1}}{\left(E_{1}-R_{1}\right)-E-i \Gamma_{E_{1}}}+\frac{4 R_{1}}{\left(E_{1}-R_{1}\right)+E+i \Gamma_{E_{1}}}\right]\right.\right. \\
& \left.\left.+\int_{-\infty}^{\infty}\left[\frac{\theta\left(E^{\prime}-E_{1}\right)}{1+\mathrm{e}^{-2 \pi z_{3}\left(E^{\prime}\right)}}-\frac{\theta\left(-E^{\prime}-E_{1}\right)}{1+\mathrm{e}^{-2 \pi z_{4}\left(E^{\prime}\right)}}\right] \frac{\mathrm{d}^{\prime} E}{E^{\prime}-E-i \Gamma_{E_{1}}}\right\}\right\},
\end{aligned}
$$

where $C_{1}$ is a constant, $E_{1}$ is the energy of the gap, $R_{1}$ is the $2 \mathrm{D}$ Rydberg energy, $\Gamma_{E_{1}}$ is the broadening parameter for both the exciton and band-to-band transition, $z_{3}(E)=\left[R_{1} / 4\left(E-E_{1}\right)\right]^{1 / 2}$, and $z_{4}(E)=\left[R_{1} / 4\left(-E-E_{1}\right)\right]^{1 / 2}$. For the $E_{1}+\Delta_{1}$ CP, Eq. (2) has $C_{1} \rightarrow C_{2}$ and $E_{1} \rightarrow E_{1}+\Delta_{1}, \Gamma_{E_{1}} \rightarrow \Gamma_{E_{1}+\Delta_{1}}$, etc. The same $2 \mathrm{D}$ Rydberg $\left(R_{1}\right)$ was used for both $E_{1}$ and $E_{1}+\Delta_{1} \mathrm{CP}$ features.

Because of their complexity, the $E_{0}^{\prime}, E_{0}^{\prime}+\Delta_{0}^{\prime}$, and $E_{2}$ features each have been described by a damped harmonic oscillator term [6 to 8, 12]. The fact that Ref. [10] has found that $E_{2}$, like the $E_{0} / E_{0}+\Delta_{0}$ and $E_{1} / E_{1}+\Delta_{1} \mathrm{CP}$ features, contains an excitonic component provides some justification in using a damped oscillator term for this feature.

Shown by the dotted curve in Fig. 1 is the fit to the experimental data using the above expressions. Since the exciton at $E_{0} / E_{0}+\Delta_{0}$ is not resolved we have taken $R_{0}(=1.6 \mathrm{meV})$ from Ref. [14]. Because of the large number of fitting parameters, values for the various gaps and their broadening parameters were initialized from values obtained by numerically taking the first-derivative of the dielectric functions with respect to energy. The details of this approach are given in Refs. [6 to 8,12]. The final obtained values of the various energies are indicated by arrows in the figures. All relevant parameters are listed in Ref. [12]. Our values of the various energy gaps, i.e., $E_{0}, E_{0}+\Delta_{0}, E_{1}-R_{1}$, $\left(E_{1}+\Delta_{1}\right)-R_{1}$, etc. are in good agreement with other works $[1,2,13,14]$. 
Table 1

Experimental and calculated values for $R_{1}$ for several different semiconductors. The latter are based on the expressions in Ref. [9]

\begin{tabular}{lcl}
\hline material & $\begin{array}{l}\text { experiment } \\
R_{1}(\mathrm{meV})\end{array}$ & $\begin{array}{l}\text { effective mass } / \mathbf{k} \cdot \mathbf{p} \text { theory } \\
R_{1}(\mathrm{meV})\end{array}$ \\
\hline $\mathrm{Zn}_{0.53} \mathrm{Cd}_{0.47} \mathrm{Se} / \mathrm{InP}$ & $\left.270 \pm 50^{\mathrm{a}}\right)$ & 300 \\
$\mathrm{CdS}$ & $\left.205 \pm 30^{\mathrm{b}}\right)$ & 290 \\
$\mathrm{CdTe}$ & $\left.145 \pm 50^{\mathrm{b}}\right)$ & 150 \\
$\mathrm{In}_{0.66} \mathrm{Ga}_{0.34} \mathrm{As}$ & $\left.92 \pm 15^{\mathrm{c}}\right)$ & 55 \\
$\mathrm{GaSb}$ & $\left.32 \pm 3^{\mathrm{d}}\right)$ & 25 \\
\hline
\end{tabular}
a) Ref. [6].
b) Ref. [8].
c) Ref. [7].
d) This work and Ref. [12].

It is important to note that due to the relatively large values of $R_{1} \quad(\approx 30$ to $300 \mathrm{meV}$ ), as listed in Table 1, the optical structure associated with the $E_{1}, E_{1}+\Delta_{1} \mathrm{CPs}$ in DZB semiconductors are actually mainly the excitonic features $E_{1}-R_{1}$, $\left(E_{1}+\Delta_{1}\right)-R_{1}$, respectively, as denoted in Fig. 1 . Almost all prior optical [1 to 5] and modulated (see, for example, [15]) optical studies have incorrectly labelled these excitonic features as " $E_{1}, E_{1}+\Delta_{1}$ ".

Our value of $R_{1}(32 \pm 3 \mathrm{meV})$ is in fairly good agreement with the effective mass/ $\mathbf{k} \cdot \mathbf{p}$ theory of Ref. [9]. According to the approach

$$
R_{1} \propto \mu_{\perp} / \varepsilon_{1}^{2}(\infty)
$$

where $\mu_{\perp}$ is the perpendicular reduced interband effective mass and $\varepsilon_{1}(\infty)$ is the high frequency dielectric function. This calculation yields $R_{1}=25 \mathrm{meV}$, as listed in Table 1 .

Table 1 also presents the experimental and calculated values of $R_{1}$ for several materials. The latter are based on the theory of Ref. [9]. The experimental numbers for $R_{1}$ exhibit a trend which is in good agreement with effective mass $/ \mathbf{k} \cdot \mathbf{p}$ theory. More reliable theoretical values can now be obtained from the recent first-principles band structure calculations which include exciton effects [10].

The solid lines in Fig. $2 \mathrm{a}$ and $\mathrm{b}$ are the experimental values of the numerical derivatives $\mathrm{d} \varepsilon_{2} / \mathrm{d} E$ for CdTe and $\mathrm{d} \alpha / \mathrm{d} E$ for GaAs, respectively, where $\alpha$ is the absorption coefficient. The former is from Ref. [8] while the latter is our SPS study. It has been shown that the SPS data in the vicinity of $E_{0}$ are proportional to $\alpha$ (see, for example, [11]). Both lineshapes are approximately Lorentzian with a slight asymmetric broadening on the high energy side. This indicates that the DE is not resolved since the firstderivative of its Lorentzian lineshape would produce an "S" shaped component. The dotted lines in Fig. 2 are least-square fits to an analytical form for the first derivative of Eq. (1), as given in Ref. [6], where $\Gamma_{0}^{\mathrm{ex}}$ has been taken sufficiently large so that the DE is not resolved. The obtained values of $E_{0}$ are indicated by arrows. The dashed lines are fits to the first-derivative of a BBSP profile, i.e., $\operatorname{Im}\left(E-E_{0}+i \Gamma_{0}\right)^{-1 / 2}$. This produces a very asymmetric lineshape which does not fit the experimental data very well.

In Ref. [6] the DE at $E_{0}$ was well resolved and it was evident that the band-to-band lineshape was BBCE and not BBSP. The results of Fig. 2 clearly demonstrate that even if the DE is not resolved the lineshape is the former and not the latter, an expression 


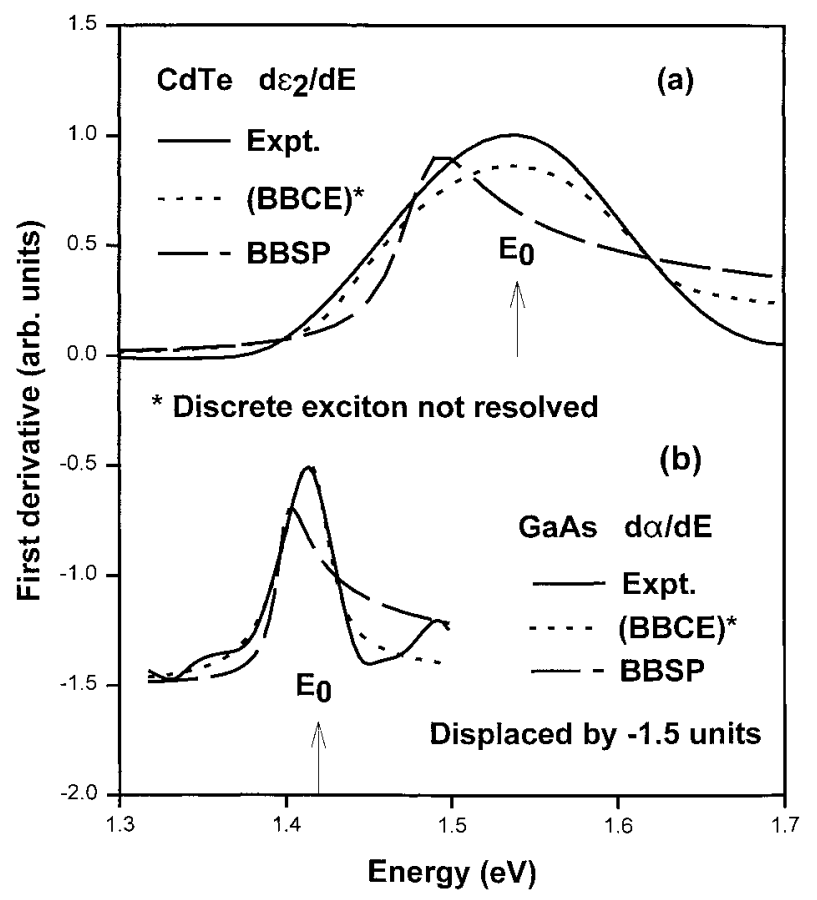

Fig. 2. The solid lines are the experimental values of a) $\mathrm{d} \varepsilon_{2} / \mathrm{d} E$ of CdTe and b) $\mathrm{d} \alpha / \mathrm{d} E$ of GaAs in the region of $E_{0}$. The dotted and dashed lines are fits to the first derivative of $\mathrm{BBCE}$ (discrete exciton not resolved) and BBSP expressions, respectively. The obtained values of $E_{0}$ from the $\mathrm{BBCE}$ fit are indicated by arrows

that has been incorrectly employed in Refs. [4,5]. Also because Adachi and coworkers and the University of Illinois-Chicago group used a BBSP expression at $E_{1}$ they were not able to extract $R_{1}$.

Acknowledgements The authors F.H.P., M.M., T.H., and K.W. thank NSF grant \#DMR-9414209, PSC/BHE grant \#666424 and the NY State Science and Technology Foundation through its Centers for Advanced Technology program for support of this project. M.M. also acknowledges support from CONACyT, Mexican Agency, through Research Project \#25135E.

\section{References}

[1] D.E. Aspnes and A.A. Studna, Phys. Rev. B 27, 985 (1983).

[2] S. Zollner, M. Garriga, J. Humlicek. S. Gopalan, and M. Cardona, Phys. Rev. B 43, 4349 (1991).

[3] P. Lautenschlager, M. Garriga, S. Logothetidis, and M. Cardona, Phys. Rev. B 35, 9174 (1987).

P. Lautenschlager, M. Garriga, and M. Cardona, Phys. Rev. B 36, 4813 (1987).

[4] S. Adachi, T. Kimura, and N. Suzuki, J. Appl, Phys. 74, 3435 (1993).

S. AdACHI and T. TAGUCHI, Phys. Rev. B 43, 9569 (1991).

K. Suzuki and S. Adachi, J. Appl. Phys. 83, 1018 (1998).

[5] C.C. Kim and S. Sivinathan, J. Appl. Phys. 78, 4003 (1995); Phys. Rev. B 53, 1475 (1996).

[6] T. Holden, P. Ram, F.H. Pollak, J.L. Freeouf, B.X. Yang, and M.C. Tamargo, Phys. Rev. B 56, 4037 (1997).

[7] T. Holden, F.H. Pollak, J.L. Freeouf, G.W. Charache, J.E. Raynolds, and C. Geller, Proc. 4th NREL TPV Generation of Electricity Conf., Denver 1998, Eds. T.J. CoutTs, J. P. BREnNer, and C.S. Allman, AIP Conf. Proc. 460, American Institute of Physics, Woodbury 1999 (p. 39). 
[8] K. Wei, F.H. Pollak, J.L. Freeouf, D. Shvydka, and A.D. Compaan, J. Appl. Phys. 85, 7418 (1999).

[9] Y. Petroff and M. Balkanski, Phys. Rev. B 3, 3299 (1971).

[10] M. Rohlfing and S.G. Louie, Phys. Rev. Lett. 81, 2312 (1998).

S. Albrecht, L. Reining, R. Del Sole, and G. Onida, Phys. Rev. Lett. 80, 4510 (1998).

[11] L. Aigouy, F.H. Pollak, J. Petruzzello, and K. Shahzad, Solid State Commun. 102, 877 (1997).

[12] M. Muñoz, K. Wei, F.H. Pollak, J.L. Freeouf, and G.W. Charache, to be published in Phys. Rev. B 60 (1999).

[13] M. Patrini, G. Guizzei, M. Galli, R. Ferrini, A. Bosacchi, S. Franchi, and R. Magnanini, Solid State Commun. 101, 93 (1997).

[14] Landolt-Börnstein, Numerical Data and Functional Relationships in Science and Technology, Vols. 17a and 17b, Eds. O. Madelung, M. Schultz, and H. Weiss, Springer-Verlag, Berlin/Heidelberg/New York 1982.

[15] M. Cardona, in: Modulation Spectroscopy, Academic Press, New York 1969.

Note added in proof: We have also recently evaluated and fit the optical constants of two samples of $\mathrm{Ga}_{1-x} \mathrm{In}_{x} \mathrm{As}_{y} \mathrm{Sb}_{1-y} / \mathrm{GaSb}(x \approx 0.16, y \approx 0.14)$. We find $R_{1}=30 \pm 5 \mathrm{meV}$ (experiment) and $26 \mathrm{meV}$ (effective mass/k $\cdot \mathbf{p}$ theory) [M. MuÑoz, K. WeI, F.H. Pollak, J.L. FreEouf, C.A. Wang, and G.W. Charache, submitted to J. Appl. Phys.]. 